\title{
The Effects of Carbon Fibre and Carbon Fibre Composite Dusts on Bronchoalveolar Lavage Component of Rats
}

\author{
Zhongyi Zhang, Xiuling WaNG, Leiguang Lin, Shudong XING, Yonghui Wu, \\ Yao LI, Lijie Wu and Baoqi GANG \\ Department of Occupational Health, School of Public Health, Harbin Medical University, China
}

\begin{abstract}
The Effects of Carbon Fibre and Carbon Fibre Composite Dusts on Bronchoalveolar Lavage Component of Rats: Zhongyi Zhang, et al. Department of Occupational Health, School of Public Health, Harbin Medical University, People's Republic of China-Carbon fibre (CF) and carbon fibre composite (CFC) as new materials have increasing industrial application. The People's Republic of China now manufactures $\mathrm{CF}$ and $\mathrm{CFC}$. This paper predicted their potential toxicity to human using bronchoalveolar lavage technique on the basis of comparisons with positive reference materials (quartz and chrysotile) and negative reference materials (titanium dioxide and alumina (SAFFIL) fibre). All rats dosed with dust showed some increase in lung weight relative to the saline control, though the only significant differences were seen between the rats dosed with quartz or chrysotile and those dosed with saline. From the morphological observation of lavage cells, a benign reaction of macrophages to $\mathrm{CF}$ and $\mathrm{CFC}$ was observed, whereas a series of changes in macrophages was involved in rats dosed with quartz and chrysotile. CF and CFC did not induce a significant increase in the total cell count or percentages of neutrophils and lymphocytes in bronchoalveolar lavage. The two materials tested had much lower toxicity than that of quartz and chrysotile, and were comparable with the effect induced by titanium dioxide and SAFFIL fibre which had minimal toxicity. The present work provides a scientific basis for the setting of occupational health standards for carbon fibre and carbon fibre composite dust in the workplace.

(J Occup Health 2001; 43: 75-79)
\end{abstract}

Key words: Carbon fibre dust, Carbon fibre composite, Bronchoalveolar lavage, Cell type

Received March 14, 2000; Accepted Oct 25, 2000 Correspondence to: $Z$. Zhang, Department of Occupational Health, School of Public Health, Harbin Medical University, People's Republic of China
Carbon fibre composite ( $\mathrm{CFC}$ ) is a new material which, by virtue of being chemically inert and having additional advantages of high strength, light weight and thermal resistance, is finding increasing industrial application. The People's Republic of China now manufactures carbon fibre $(\mathrm{CF})$ and $\mathrm{CFC}$. During the manufacturing and processing of both CF and CFC, because dust may be released into the workplace air, there is an understandable concern that these processes may present an occupational risk $^{11}$. Because of the short history of production of both $\mathrm{CF}$ and CFC, epidemiological investigation could provide only limited confidence that there were no potential adverse effects. Bronchoalveolar lavage is a well established technique for predicting the toxicity of various dusts in animals. We report a study in which this technique is used to examine the in vivo cytotoxicity of $\mathrm{CF}$ and CFC dust samples as an aid to predicting their potential human hazard.

\section{Materials and Methods}

Rats

Twenty-one male and twenty-one female Wistar strain rats (weight range $200-300$ g) were randomly assigned to seven test groups ( 6 in each group; 3 of each sex per group).

\section{Test samples}

The CF sample used was obtained from a factory in $\mathrm{Ji}$ Lin City of the People's Republic of China producing PAN-based carbon fibre. A bundle of carbon fibres ( 3 $\mathrm{cm}$ in length) was packed into a container filled with distilled water. The whole was then frozen at $-80^{\circ} \mathrm{C}$. The ice embedded CF blocks were cut along the long axis of the fibres with a microtome. The sections were allowed to thaw and the water was evaporated to release residual CF. These were then sieved through a $65 \mu \mathrm{m}$ (250 mesh) sieve. Microscopic analysis showed that the product had fibre diameters in the range of $6-8 \mu \mathrm{m}$ with 
a median length of $37.5 \mu \mathrm{m}$. 97\% of the fibres were $\leq 200 \mu \mathrm{m}$ long. CFC was obtained from an aircraft factory in Harbin City of the People's Republic of China. A coarse sample was produced by drilling the composite. This sample was then further reduced by grinding in an electrically operated agate pestle and mortar to produce a respirable fraction. $93 \%$ of the particles in the final product were $<5 \mu \mathrm{m}$ in diameter.

\section{Control samples}

Quartz and chrysotile were used as positive controls and $\mathrm{TiO}_{2}$ and SAFFIL fibre as negative controls. A saline control was also included.

A sample of quartz was obtained from the Institute of Occupational Medicine, Chinese Academy of Preventive Medicine. The sample was analysed as $98 \%$ silica and $99 \%$ of particles were $<5 \mu \mathrm{m}$ in diameter. A sample of the UICC chrysotile A standard international reference sample was supplied by Dr. J.M.G. Davis, Institute of Occupational Medicine, Edinburgh, UK. Titanium dioxide is a commercially supplied commodity reagent (analytical grade) from Beijing Chemical Plant. The sample was ground to a fine powder in an electrically operated agate pestle and mortar. $96 \%$ of the particles in the ground sample were $<5 \mu \mathrm{m}$ in diameter. SAFFIL fibres in an inorganic refractory fibre consisted of $95 \%$ alumina with $4.3 \%$ silica and a few trace impurities. A ground sample was supplied by Dr. G.H. Pigott, Central Toxicology Laboratory, Imperial Chemical Industries plc, UK. The fibres supplied have a median diameter of 3.2 $\mu \mathrm{m}$ with $98 \%<5 \mu \mathrm{m}$ in diameter. The fibres have a median length of $62 \mu \mathrm{m}$ with $95 \%<211 \mu \mathrm{m}$.

\section{Preparation and administration of dust suspensions}

Saline was the vehicle for all dusts. Suspensions were prepared by manual shaking. All test samples, except chrysotile, were prepared at $50 \mathrm{mg} / \mathrm{m} /$. Chrysotile was prepared at $12.5 \mathrm{mg} / \mathrm{m} l$ because of the mortality associated with the higher dose. The suspensions were sterilised by autoclaving at $120^{\circ} \mathrm{C}$. Just prior to administration penicillin was added at a concentration of $400 \mathrm{iu} / \mathrm{m} /$. The samples were administered by intratracheal injection under light anaesthesia. The volume of suspensions, including saline, administered was $1 \mathrm{~m} /$ for each animal, which contained $50 \mathrm{mg}$ different samples (12.5 $\mathrm{mg}$ for chrysotile sample), respectively, for the groups.

\section{Brochoalveolar lavage}

One month after administration all the animals were anaesthetised with $2 \%$ pentobarbital and sacrificed by exsanguination via the abdominal aorta. The thorax was opened, the left primary bronchus ligatured and the right lung lavaged four times with approximately $2.5 \mathrm{ml}$ physiological saline. External massage was used to ensure recovery of cells in the airways; $10 \mathrm{~m} l$ of lavage fluid was collected from each animal.

\section{Post mortem examinations}

For observation, lavage fluid cells were recovered by centrifugation at $2,000 \mathrm{rpm}$ for $10 \mathrm{~min}$. Cell pellets were resuspended in physiological saline. Total cell counts were performed in a haemocytometer. Aliquots were also sedimented in a cytocentrifuge and stained with Giemsa for differential cell counts.

The left lung of each rat was removed and weighed.

\section{Statistical treatment of results}

Intergroup comparisons were made by analysis of variance with SYSTAT software, comparisons were by Duncan's test with a $P$ value $<0.05$ considered significant.

\section{Results}

Figure 1 shows the intergroup comparison of the wet weight of the left lungs. All rats dosed with dust showed some increase in lung weight relative to the control, though the only significant differences were between the rats treated with quartz or chrysotile and those treated with saline. As similar results were observed when the wet lung weight was expressed as a percentage of the rat body weight, this effect does not reflect differences in the size of the animals concerned.

An increase in the size of particle containing cells was evident under the optical microscope for rats treated with carbon fibre or SAFFIL fibres, but the cell membranes remained intact. Some fibres were apparently phagocytosed by 2 or more macrophages, with the longer fibres sometimes giving the appearance of a string of beads. This was more remarkable in the rats treated with $\mathrm{CF}$. In rats treated with $\mathrm{CFC}$ and $\mathrm{TiO}_{2}$ black dust, particles within macrophages were seen in the bronchoalveolar lavage fluid. In some instances the macrophage nucleus was not easy to identify due to the large number of particles in the cell. Even in such particle laden cells the outer membrane remained visually intact.

In rats treated with quartz macrophages some had one or more of a series of morphological changes such as enlarged size, poor staining of the cytoplasm and nucleus, cytoplasmic vacuolation and even apparent enucleation. In addition cell debris and fragments could be seen in the lavage fluid indicating cellular disruption. In rats treated with chrysotile, similar changes were observed but to a lesser extent.

Figure 2 shows the results of total cell counts. The maximum cell count was obtained from rats treated with quartz, followed by that in rats treated with chrysotile. Cell counts in the other groups were much lower. Rats treated with $\mathrm{TiO}_{2}$ or SAFFIL had counts slightly lower, but none of these changes attained statistical significance. 


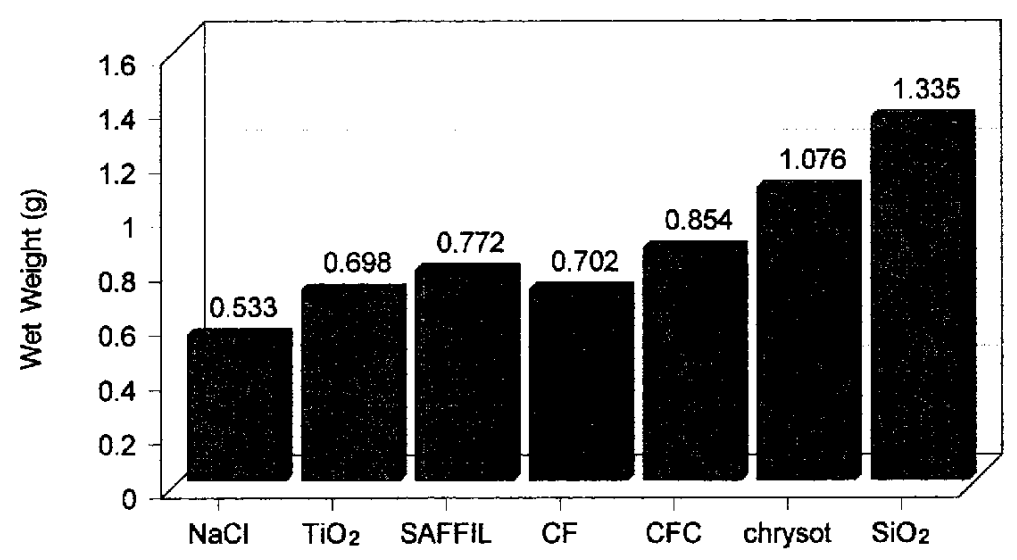

Fig. 1. The intergroup comparison of the wet weight of the left lungs. $\mathrm{NaCl}$ : saline, $\mathrm{TiO}_{2}$ : titanium dioxide, $\mathrm{SAFFIL:} \mathrm{inorganic} \mathrm{refractory} \mathrm{fibre,} \mathrm{CF}$ : carbon fibre, CFC: carbon fibre composite, chrysot: UICC chrysotile, $\mathrm{SiO}_{2}$ : quartz. All rats dosed with dust had some increase in lung weight compared to rats dosed with saline. Only significant differences were seen between rats dosed with quartz or chrysotile and those with saline.

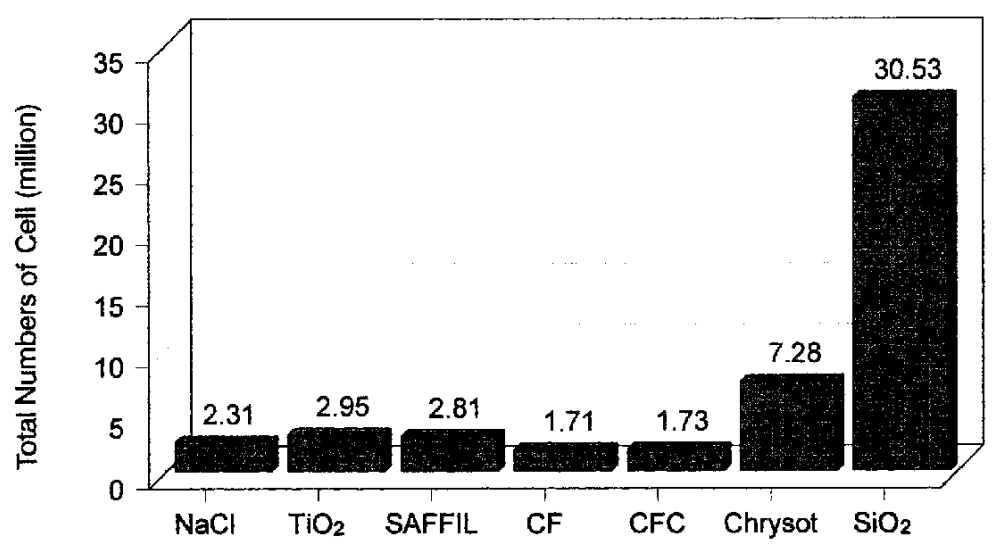

Fig. 2. The intergroup comparison of total cell counts. $\mathrm{NaCl}$ : saline, $\mathrm{TiO}_{2}$ : titanium dioxide, SAFFIL: inorganic refractory fibre, CF: carbon fibre, $\mathrm{CFC}$ : carbon fibre composite, chrysot: UICC chrysotile, $\mathrm{SiO}_{2}$ : quartz. The highest cell count was seen in rats dosed with quartz, followed by that in rats dosed with chrysotile. Cell counts in other groups were much lower.

Differential cell counts are shown in Fig. 3. In most of test samples macrophages were the predominant cell type, accounting for $92.6 \%-98.8 \%$ of cells counted in all groups except those dosed with quartz or chrysotile. Statistical analysis showed the proportion of macrophages was significantly reduced in rats treated with quartz $(53 \%)$ and chrysotile (76.3\%) compared with those treated with other test samples.

The percentage of neutrophils reflected the differences seen in macrophages in the quartz group $(41.75 \%)$ and the chrysotile group $(22.0 \%)$, both significantly higher than controls. There were no other significant intergroup differences. The percentage of lymphocytes $(5.25 \%)$ in the quartz group was significantly above controls. The only other group to exceed $1 \%$ was chrysotile $(1.5 \%)$, though the difference was not statistically significant. Analysis of the absolute values for each of the cell types examined confirmed the increase in those treated with quartz. In rats treated with chrysotile the absolute values for each cell type were also increased compared to the other groups (excluding quartz) but the changes did not attain statistical significance. There were no other significant intergroup differences. 


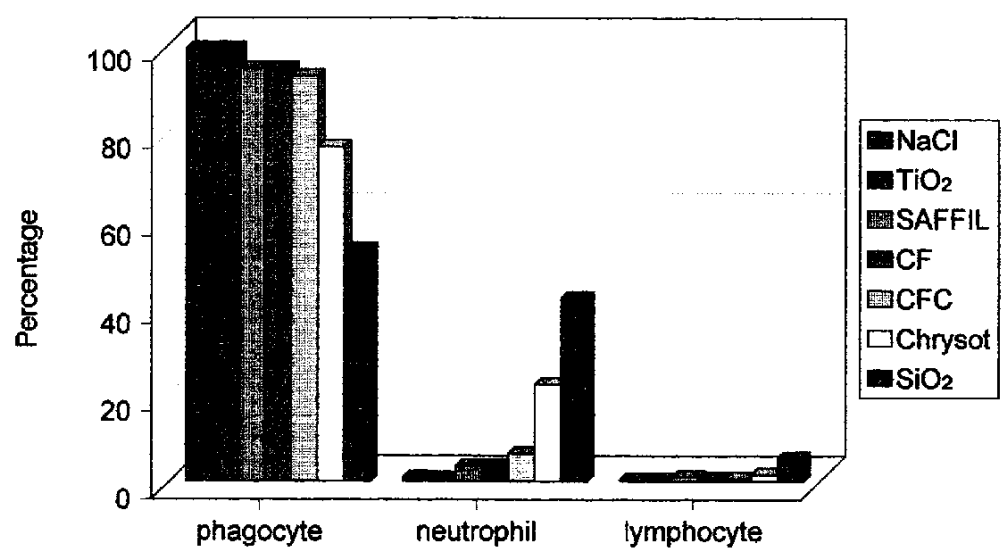

Fig. 3. The intergroup comparison of counts of the various cells in bronchoalveolar lavage. $\mathrm{NaCl}$ : saline, $\mathrm{TiO}_{2}$ : titanium dioxide, SAFFIL: inorganic refractory fibre, CF: carbon fibre, CFC: carbon fibre composite, chrysot: UICC chrysotile, $\mathrm{SiO}_{2}$ : quartz. In most of the test samples macrophages were the predominant cell type, accounting for $92.6 \%-98.8 \%$ of cells counted in all groups except those dosed with quartz $(53 \%)$ or chrysotile $(76.3 \%)$. The percentages of neutrophils in rats dosed with quartz $(41.95 \%)$ and chrysotile $(22.0 \%)$ were significantly higher than in rats dosed with $\mathrm{TiO}_{2}$, SAFFIL fiber or saline. The percentage of lymphocytes in rats dosed with quartz $(5.25 \%)$ was significantly increased, and in rats dosed with chrysotile (1.5\%) was higher but to a lesser extent.

\section{Discussion}

The two samples tested in this study have different physical properties. CF was in fibrous form whereas the CFC sample was a non fibrous particulate. For this reason different control materials were selected for more relevant comparisons. Quartz and chrysotile are the two most widely accepted standard references as positive controls in studies of cytotoxicity and fibrogenicity. $\mathrm{TiO}_{2}$ is also generally accepted as the standard inert particulate showing little or no cytotoxicity and fibrogenicity in a number of studies ${ }^{2}$. SAFFIL fibres were designed to minimise biological activity and their inert nature has been confirmed in a number of experiments both in vivo and in vitro ${ }^{33}$.

On the basis of the lung wet weight only the animals treated with quartz and chrysotile could be considered to show a significant reaction to dust. The slight increase in wet lung weight in the animals treated with CF, CFC, $\mathrm{TiO}_{2}$ and SAFFIL over those treated with saline alone is too small to be considered significant, but it should be noted that the wet lung weight in rats treated with either CF or CFC was slightly below that recorded for the negative control samples.

From the morphological observation of lavage cells it was evident that the dusts persisted in the lung for at least one month after injection, but CF and CFC dusts were apparently phagocytosed by macrophages and the
CF or CFC laden macrophages were morphologically intact and showed no evidence of an adverse reaction to the dust content. Similar changes were seen with $\mathrm{TiO}_{2}$ dust and SAFFIL fibres and are considered typical of biologically inert dust. By contrast both quartz and chrysotile resulted in obvious morphological changes in macrophages and by virtue of the cellular debris seen could be considered to be cytotoxic to the phagocytic cells.

In previous studies total and differential counts of lavage cells have been used as indices to evaluate the toxicity of dusts to the lung. An increase in the total number of cells in lavage fluid has been observed in animals treated with various insoluble dusts ${ }^{4}$. From our observations the total numbers of cells in rats treated with $\mathrm{CF}$ or CFC dust were significantly smaller than those seen with quartz or chrysotile and comparable with the negative controls used. This is considered to confirm the very weak nature of the biological response to $C F$ and $\mathrm{CFC}$ dust.

The only test samples to show significant changes in differential cell counts were quartz and chrysotile. In rats treated with either of these dusts, the percentage of macrophages was significantly lower than that for the other test samples and control. This mainly reflected an increase in the number of neutrophils in the lavage fluid. The presence of neutrophils in lavage fluid is believed to be a marker of pulmonary fibrosis and is considered a 
sensitive indicator of the acute effects of dust on the lung). It is suggested that this influx into the alveolar spaces reflects cell injury. In this context the lack of any significant increase in neutrophil levels in rats treated with CF or CFC is further evidence of the inert nature of these materials.

Significant increases in the number of lymphocytes occurred only in rats treated with quartz. There was no increase in the number of lymphocytes in rats treated with CF or CFC. This observation is in accord with that of Martin et al. ${ }^{(6)}$ in which five carbon fibre composite samples and one glass fibre composite sample were compared to quartz by using the bronchoalveolar lavage technique. These authors found that three of these five composite samples showed consistently low toxicity but two provoked an increase in alveolar macrophages and caused significant accumulation of neutrophils in rat lungs, but they were all much less toxic than quartz. This is in accord with the inhalation test performed by Owen et al. who exposed rats to polyacrylnitrile based carbon fibres $\left(20 \mathrm{mg} / \mathrm{m}^{3}\right)$ for $16 \mathrm{wk}(6 \mathrm{~h} / \mathrm{d}, 5 \mathrm{~d} / \mathrm{wk})^{7}$. The only histological finding in the lungs was the accumulation of alveolar macrophages. These findings agree well with observations in the short term tests, though the size of the fibres tested suggests that respirability in the rat (and therefore the lung burden) would be low.

The present investigation shows that in vivo toxicity of the $\mathrm{CF}$ and $\mathrm{CFC}$ dust samples tested was comparable to materials of known low biological activity and distinct from that caused by quart $z$ or chrysotile. It should be noted that no clear difference in toxicity was demonstrated between CF and CFC. Whereas the fibre is almost pure carbon, the composite uses a binding agent to trap the carbon fibre filaments, so that the chemical composition of CFC dust is more complicated. The fact that the biological effects of the two dusts showed no significant difference indicates that in this instance the binding agent did not influence the toxicity of the composite.

This investigation provides some experimental evidence for the setting of occupational exposure limits for $\mathrm{CF}$ and CFC dusts. Based on the in vivo cytotoxicity data available in this study, the maximum allowable concentrations of carbon fibre and carbon fibre composite dusts in the air of the workplace would be similar to that for $\mathrm{TiO}_{2}$ dust.

Acknowledgments: The authors wish to thank Dr. G.H. Pigott for rewriting this manuscript and for helpful critical comments on the work.

\section{References}

1) Jones HD, Jones TR, Lyle WH. Carbon fiber: Results of a survey of process workers and their environment in a factory producing continuous filament. Ann Occup Hyg 1982; 26: 861-868.

2) Environmental Health Criteria 24. Titanium. Geneva: World Health Organization, 1982: 39-42.

3) Pigott GH, Ishmael J. A strategy for the design and evaluation of a 'safe' inorganic fibre. Ann Occup Hyg 1982; 26: 371-380.

4) Brain JD. The effects of increased particles on the number of alveolar macrophages. In: Inhaled Particle III. London: Unwin Brothers Limited, 1971: 200-233.

5) Morgan A, Moores SR, Holmes A, Evans JC, Evans NH, Black A. The effect of quartz, administered by intratracheal instilation, on rat lung, I. The cellular response. Environ Res 1980; 22: 1-12.

6) Martin TR, Meyer SW, Luchtel DR. An evaluation of the toxicity of carbon fiber composites for lung cells in vitro and in vivo. Environ Res 1989; 49: 246-261.

7) Owen PE, Glaister JR, Ballantyne B, Clary JJ. Subchronic inhalation toxicology of carbon fibers. J Occup Med 1986; 28: 373-376. 